\title{
Geometric Patterns, Light and Shade: Quantifying Aperture Ratio and Pattern Resolution in the Performance of Shading Screens
}

\author{
Niloufar Emami ${ }^{1} \cdot$ Harry Giles ${ }^{1}$
}

Published online: 4 January 2016

(C) Kim Williams Books, Turin 2015

\begin{abstract}
This research focuses on the application and performance assessment of geometric patterns as shading screens and shows how the geometric patterns can function as a design agency, an environmental control system, and a cultural element. We begin with a brief review of the underlying rules of creating two-dimensional geometric patterns, and then look at how these patterns evolve as threedimensional shading screens in buildings. We next discuss a predictive model for translating complex patterns to simple patterns concerning their perforation ratio, granularity, and morphology. This is followed by an experimental and simulation study for measuring the daylighting performance of some simple shading screens. The result of this phase assesses the agreement among experimental and numerical studies. Finally, we evaluate the performance of a screen inspired by a Persian pattern.
\end{abstract}

Keywords Geometric patterns - Persian patterns - Shading screens - Solar screens · Predictive model · Perforation ratio · Granularity · Daylighting performance

Niloufar Emami

nemami@umich.edu

Harry Giles

hgiles@umich.edu

1 A. Alfred Taubman College of Architecture and Urban Planning, University of Michigan, 2000 Bonisteel Boulevard, Ann Arbor, MI 48109-2069, USA 


\section{Introduction}

Islamic art has three main expressions: calligraphy, floral ornament and geometric ornament (Cenani et al. 2007). In keeping with religious beliefs, Persian art avoided anthropomorphic forms, and instead expanded geometric explorations which resulted in an extraordinary, large, complex and elegant collection of periodic patterns (Abas and Salman 1992).

In architecture, geometric patterns were mostly applied in religious buildings and tombs, but also in some residential buildings. In that case, the frequency and level of complexity of the patterns were related to the wealth of the owner. These patterns were applied to flat surfaces, as well as curved surfaces (Fig. 1).

Geometric patterns were also applied to perforated screens. Daylight penetrates into the spaces through perforated screens that allow light to filter into space. Such screens are not merely ornamental; their environmental performance is also significant. The goal of this present research is to study how different geometric patterns are realized in the shading screens, and the degree of daylighting performance that they provide for architectural spaces. This paper looks at some rules for creating geometric patterns. Next it focuses on the application of these patterns in creating three-dimensional shading screens. Then it discusses a predictive model on how to translate complex patterns to simple patterns. After that, it studies the experimental point-in-time daylighting illuminance in a room considered as the base case, while different shading screens are installed in front of its sole south faced window. The experimental measurements are then compared to simulations carried out by DIVA for Rhino (Jakubiec et al. 2011) and Flamingo nXt plugin for Rhino (http://nxt.flamingo3d.com), in order to assess the agreement among the outcomes. The results provide a ground for expanding the research in simulation-based performance assessment for future designs of shading screens inspired by geometric patterns. Finally, it assesses the performance of a screen that is designed inspired by a Persian pattern with different resolutions.
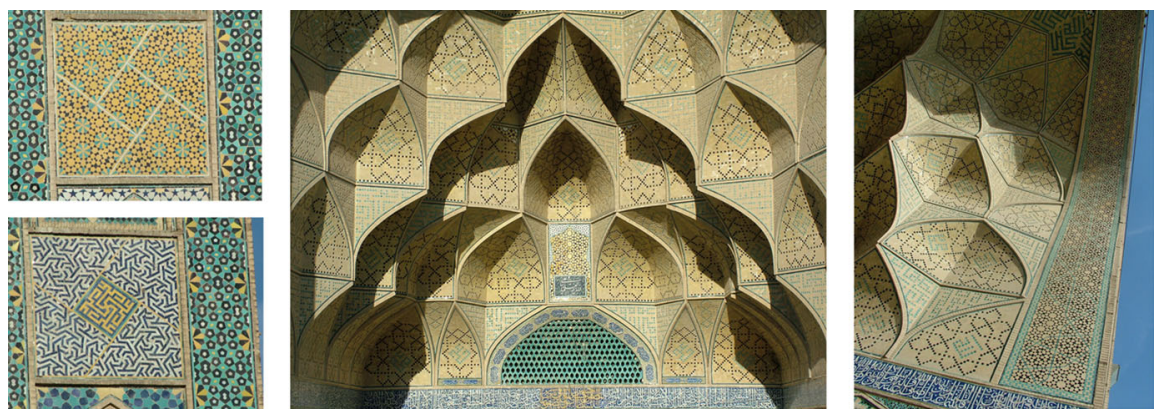

Fig. 1 Geometric patterns on flat and curved surfaces, Jamé Mosque of Isfahan 


\section{Two-Dimensional Patterns: Mathematical Construction of Geometric Patterns}

According to Syed Jan Abas and Amer Shaker Salman, "the overall impression that is created is that from the earliest of times, the inventors of Islamic patterns were dedicated-geometers inspired by theoretical compass/ruler based constructions of the classical Greek geometry. No thought or credit has been given to the practical experience of tiling with real shapes" (Abas and Salman 1992:44). With this in mind, they divide patterns into four different categories, the first two of which can be purely discovered through practical experience of tiling with simple shapes, while the last two refer to some geometrical construction rules. We will briefly summarize these here.

The first category encompasses the patterns based on single-shaped tiles. In this case, just one single tile is used and through varying the orientation of neighboring pieces, or through removing pieces, related design emerges (Fig. 2). It is worth noting that Abas and Salman are not referring to monohedral tessellations (one face tiling) since the gaps between tiles are suggesting tiling with two different shapes. We believe instead they are referring to the experience of constructing patterns by propagating one geometric shape, thus other shapes may appear in the gaps or holes. Looking specifically at a case study, namely the Shaykh Abd al-Samad Shrine complex in Natanz, Iran, there are examples of using a ceramic tile of one geometric shape over flat surfaces of plaster and mud or in combination with brick.

The second category points to the experience of storing and stacking simple tiles, leading to experimentation with tiles that overlap. We believe that this incident might have been a source of inspiration to design and make complicated unit patterns. Figure 3 shows how a square tile when rotated $45^{\circ}$ and placed on top of an identical tile gives rise to an eight-pointed star shape.

The third category indicates patterns that evolve when some geometrical construction is added to familiar shapes such as a square (Fig. 4). The fourth category refers to patterns based on concealed grids which include a general procedure as follows: "a grid of some sort is drawn and then polygons/circles are placed in some regular fashion. Then the circumferences of figures are also divided, marked with points and joined together. After the pattern emerges, the construction lines are removed. The lines in the pattern are often replaced with interlacing lines to enhance the effect" (Abas and Salman 1992: 46) (Fig. 5).

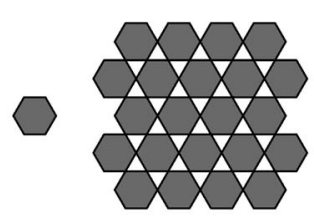

propagation a

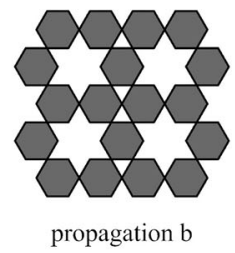

propagation $b$

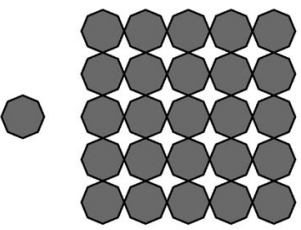

propagation c

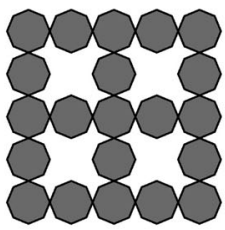

propagation $\mathrm{d}$

Fig. 2 First category of patterns based on single shaped tiles, after (Abas and Salman 1992) 


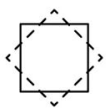

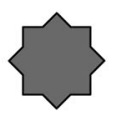

overlaying tiles creating a new tile

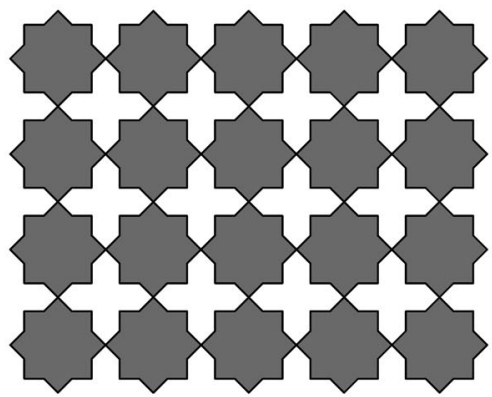

propogation

Fig. 3 Second category of patterns with overlapping tiles, after (Abas and Salman 1992)
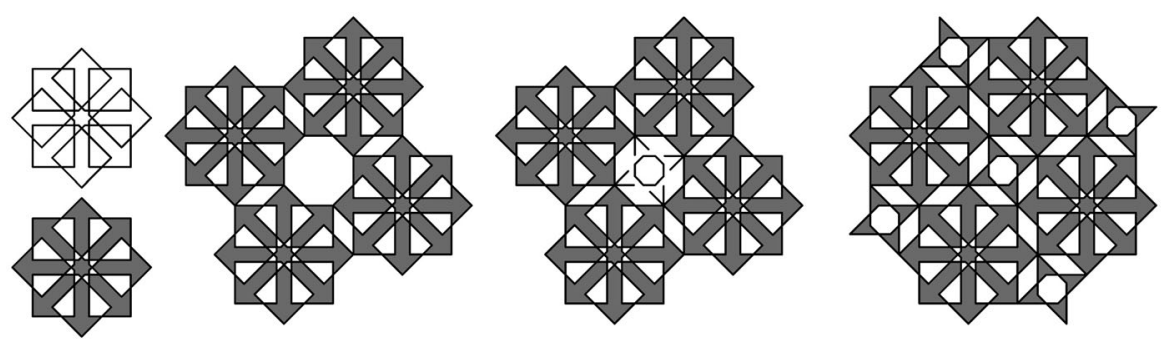

Fig. 4 Third category of patterns based on geometric construction, after (Abas and Salman 1992)
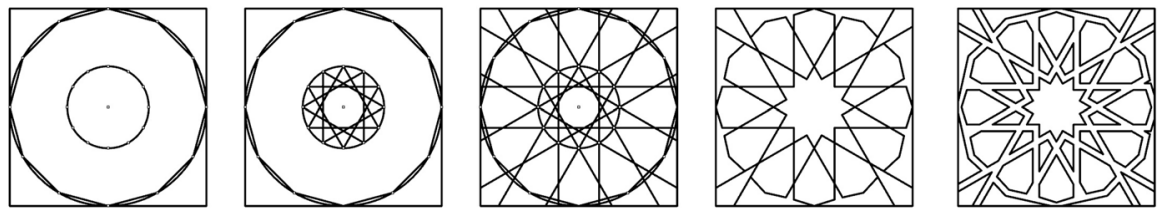

Fig. 5 Fourth category of patterns based on concealed grids, after (Abas and Salman 1992)

Reviewing these four categories, we see that they are either based on practical experience of tiling (rather than the knowledge of mathematics) or based on a very generalized and sometimes vague method of geometric construction. There are other approaches in categorizing geometric patterns with a more scientific base. For instance, Reza Sarhangi (2012) describes four methods of tiling constructions: "Radial Grid method", "polygons in contact method (PIC)", "star polygons" and "modularity" (Sarhangi 2012). It is worth noting that he mentions modularity as a method that provides the layout of a tessellation which is used for conceptualization, and does not necessarily involve the making of the individual tiles. In another study, Eric Broug (2013) categorizes the geometric ornamentation patterns into three main families which include most (but not all) of the patterns. The geometric families are 


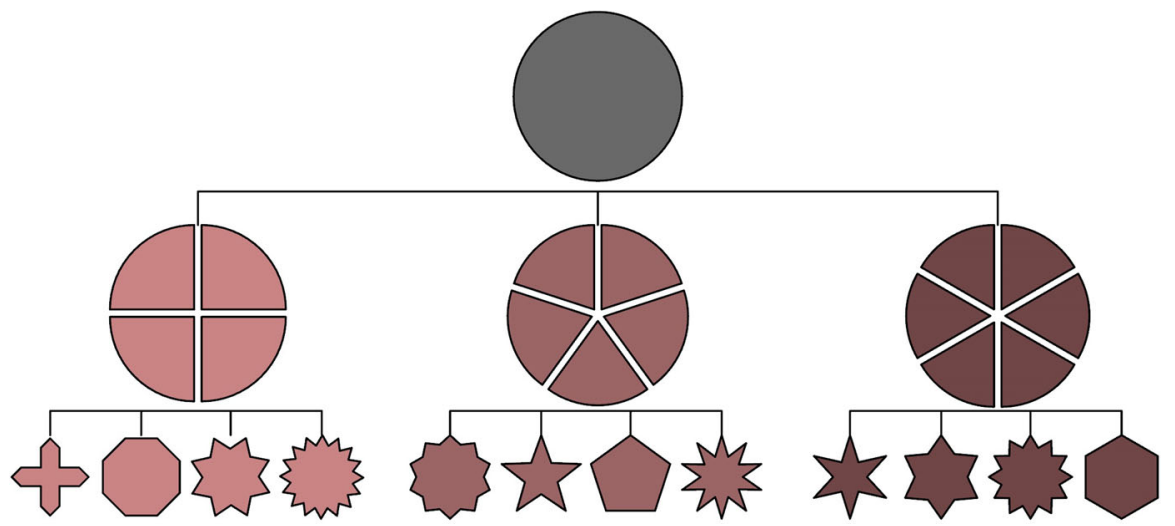

Fig. 6 Family of geometric patterns, after (Broug 2013)
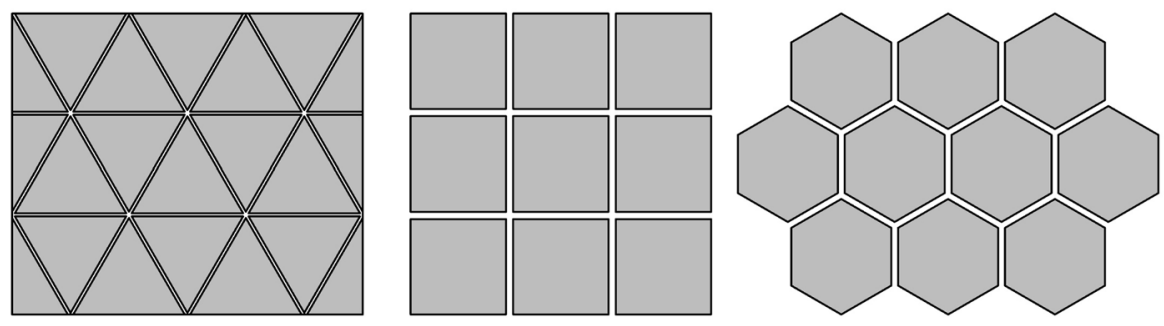

Fig. 7 Grids as the invisible structure of patterns, after (Broug 2013)

categorized according to the number of sections into which a circle has been divided in order to create the pattern. If the circle is divided into four, five or six equal parts, it is called four-fold, five-fold and six-fold designs respectively (Fig. 6). Broug situates 12-fold designs within the family of six-fold designs. It is noticeable that seven-pointed or eleven-pointed stars are not part of any of the categories represented in the geometric family tree. In addition, grids are suggested to provide a structure of polygons and act as hidden structures for arranging the patterns (Fig. 7).

\section{Three-Dimensional Perforated Screens: How Patterns Evolve as Screens}

There is a close connection between geometric patterns and the design of shading screens, as the two-dimensional patterns that were identified by color and material in tiling evolve into three-dimensional screens that function as daylight control systems. As previously stated, one objective of this present research is to study how the patterns are realized as a perforated panel. These external perforated panels, fixed in front of windows, are called "shading screens", or sometimes "solar 


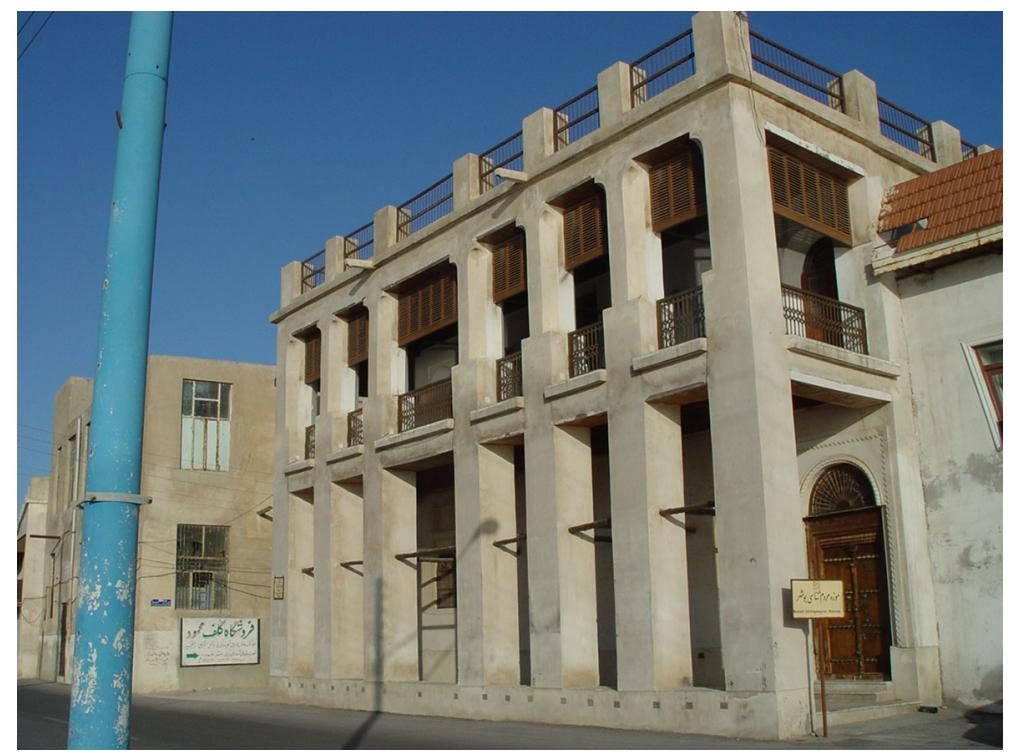

Fig. 8 Shanāsheel, Taheri House, Bushehr, Iran (photo: Anahita Khodadadi, reproduced by kind permission)

screens". They allow natural daylighting to enter the building while controlling thermal discomfort and the incidence of glare. They resemble a traditional solution named Mashrabeya or Rawshan in Middle Eastern countries, which are made of wooden lattices (Sherif et al. 2012b).

In Iran, one historical example of the use of shading screens is the wooden balcony known as Shanāsheel that provides privacy. In tropical regions of Iran, especially in Bushehr city, Shanāsheel plays a role in providing natural ventilation for the buildings (Fig. 8).

Another shading screen in Iran is called Shobāk (or Shabake), which is an unglazed latticework made of wood, brick or stone. The important feature of Shobāk is that in addition to providing daylight control, view, space privacy and the reduction of glare, they allow ventilation for space (Fig. 9).

In one study, external perforated solar screens for daylighting were evaluated by simulation in order to identify the minimum perforation percentage (Sherif et al. 2012b). The illuminance levels in different zones of the room (near window, in the middle of the room and far from window) were evaluated and a non-uniform distribution of perforations within the screen was suggested. In addition, different perforation ratios was offered based on the orientation of the window. Nermine Abdel Gelil (2006) studied the use of traditional Islamic latticework named Mashrabiyya and the Japanese Machiya-goshi and offered a new alternative for apartment buildings of contemporary Cairo, allowing residents to adjust the degree of privacy, airflow and lighting. In another study (Emami et al. 2014), a concrete shading panel was designed parametrically, and its structural and daylighting performance was assessed simultaneously using genetic algorithms. It appears that these patterns have a great potential for use as shading screens in different cultural contexts. 

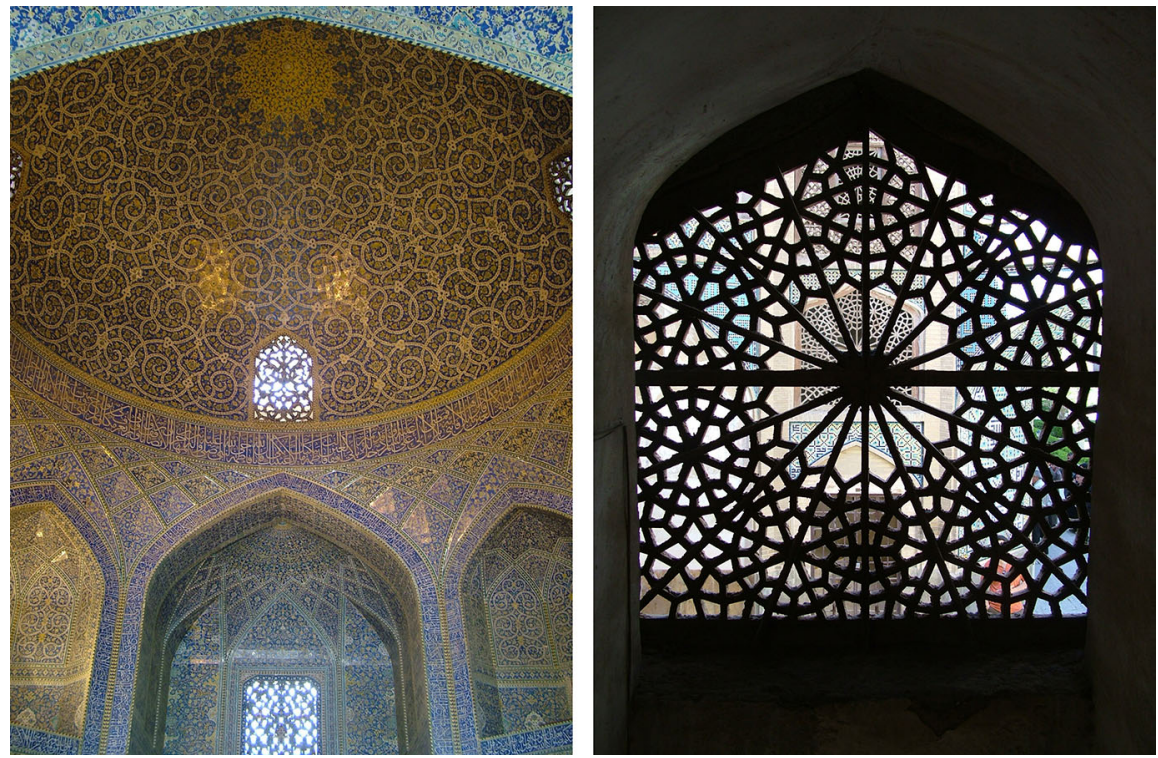

Fig. 9 Shobāk, "CharBaagh" school, Isfahan

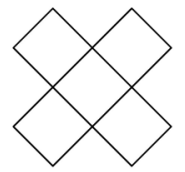

Plain

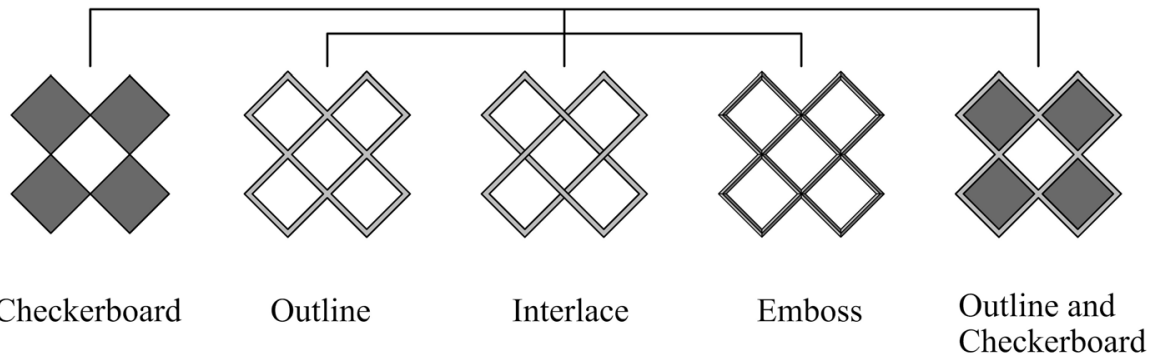

Fig. 10 Rendering styles of plain geometric patterns, after (Kaplan 2000)

\section{Perforated Geometric Patterns as Screens}

As Craig Kaplan (2000) mentions, the output of the construction process of geometric patterns is a planar graph that was never drawn as lines but realized with mosaic of small terracotta tiles, or carved into wood or stone to build trellises and latticework. He categorized different renderings of geometric patterns into six categories: plain, checkerboard, outline, interlace, emboss, and outline-and-checkerboard (Fig. 10). 
Building on Kaplan's categories, this section studies how perforated screens can be realized with different renderings. This is regardless of material and craft methodologies, and only addresses different possible realizations of plain geometric patterns. For this purpose, a simple plain pattern (Fig. 11a) is selected and then rendered into different possible realizations (Fig. 11b-d). The white color indicates the voids, whereas the gray color indicates the solids. Different shades of gray are utilized as an aid in representation and represent an infill material. In realizing a shading screen with the same pattern, if a configuration is simply outlined, embossed or is interlaced, the perforation ratio in all cases is the same. Therefore in this study, they are all categorized within the "outline" category.

The first category of rendering plain patterns is the "checkerboard" rendering, in which specific geometric shapes that have been propagated through the pattern are filled while other shapes are left empty. This can be constructed either by putting bricks or stone pieces on top of each other (Fig. 12), or by carving a pattern out of wood. It should be noted that the focus of this categorization is neither the material nor the crafts process, but how the two-dimensional geometric patterns are translated into three-dimensional screens and then perceived spatially.

The second approach is to offset the lines that create the geometric pattern and then to perforate all the generated shapes among the lines. Following this logic, the screen may be carved out of a wooden panel or made of stone parts (Fig. 13). Although these craft methods are fundamentally different, the way a specific pattern
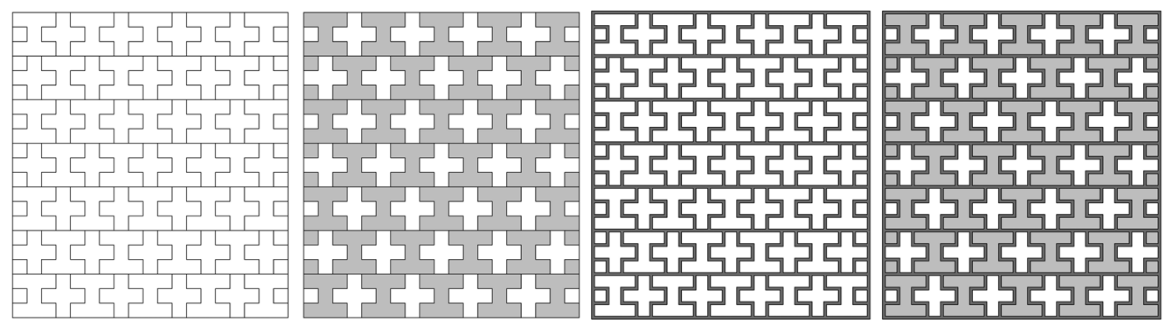

Fig. 11 Model illustration, (from left to right): a plain, b checkerboard $\mathbf{c}$ outline, d outline-andcheckerboard
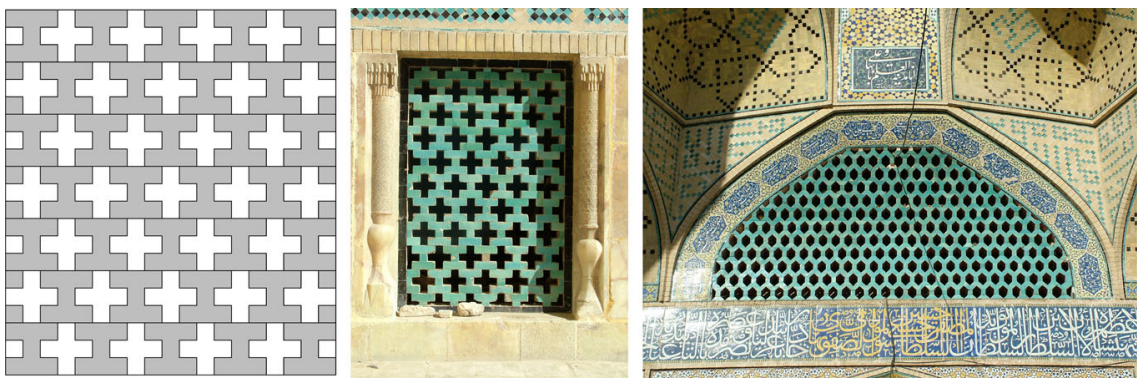

Fig. 12 From two-dimensional to three-dimensional: left checkerboard pattern; middle and right screen in Ostad Ivan, Jamé Mosque of Isfahan, Iran 

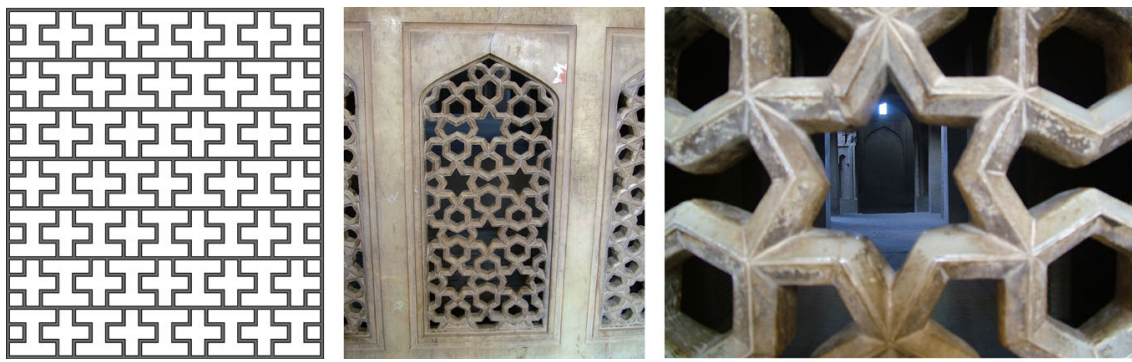

Fig. 13 From two-dimensional to three-dimensional: left outlined illustration; middle and right screen in Darvish Ivan, Jamé Mosque of Isfahan, Iran
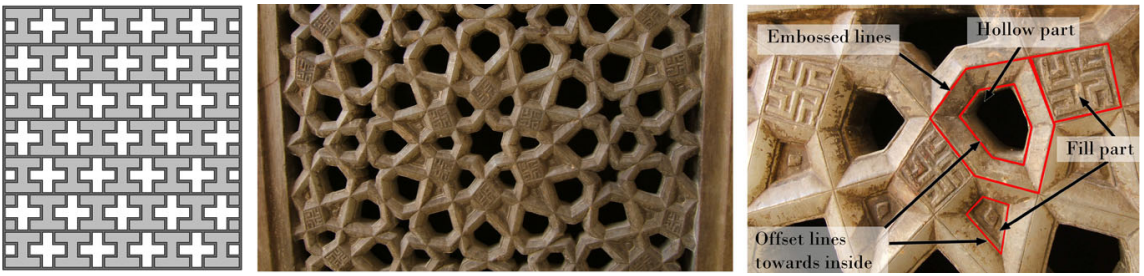

Fig. 14 From two-dimensional to three-dimensional: left outlined and checkerboard illustration; middle screen in Saheb Ivan, Jamé Mosque of Isfahan, Iran; right a close-up showing filled/hollowed pattern

is perceived under light and shade is nearly the same, which is the focus of this study.

The third category of realization, outline-and-checkerboard, employs the outline method (meaning that all the lines are offset) and then the checkerboard method to fill some (but not all) of the geometric patterns. In Fig. 14, dark gray colors represent the embossed lines, whereas the light gray color represents the infill material. It should be noted that the embossed outlines are an aid to emphasize on some of the underlying lines that created the pattern to make the original pattern recognizable. In other words, if the lines were simply outlined and not embossed, some smaller patterns would not have been recognizable, and would be conceived as a larger geometric shape. This is illustrated more clearly in Fig. 16. All the methods mentioned above can be design strategies for creating different perforation ratios based on the program of the space and the preferred lighting levels.

In order to illustrate the effect of embossed lines in aiding pattern recognition, a sample pattern referred to as a "square-based twelve-pointed star" in (Sarhangi 2012) has been selected (Fig. 15a) to be translated into a hypothetical screen. The embossed lines are drawn in light-grey, along with offset lines towards inside and outside (drawn in dark gray) to create a sample shading pattern (Fig. 15b). Finally, different carving patterns are illustrated in Fig. 16, with and without the presence of the embossed lines represented by light gray. It is noticeable that as fewer modules are carved out, the recognition of the original pattern becomes more challenging in cases without the embossed lines. 

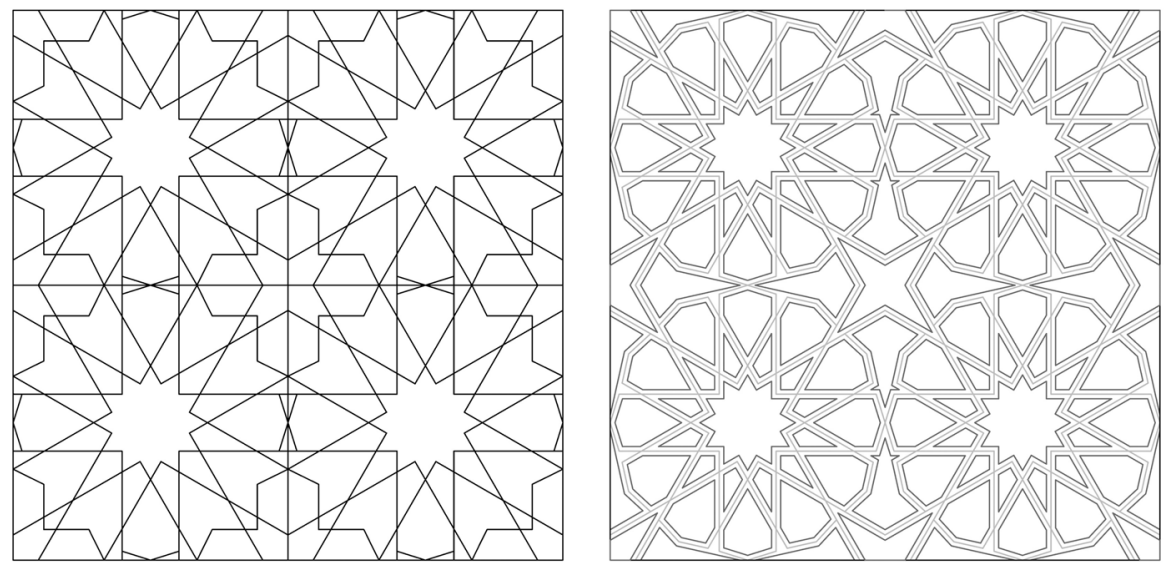

Fig. 15 a Left square-based twelve-pointed star, after (Sarhangi 2012); b right hypothetical shading screen

\section{Geometric Patterns with Glass Infill}

Another approach for realizing geometric patterns is to use them in the same layer in which the glass is installed. In this case, the patterns are used as outlined frames to hold the infill glass. Persian patterns are filled with either clear or colored glass. The light passing through the colored glass reflects the geometric patterns on to the wall and ground with a colored shadow. This approach creates a unique architectural space (Fig. 17).

\section{Superimposed Geometric Patterns}

Last, but not least, is the process of superimposing layers of geometric patterns. In this case, the final pattern is less recognizable, and creates unpredictable shadow patterns that change during different times of the day and throughout the year. The Louvre Abu Dhabi Museum, which is a new art museum in the United Arab Emirates, has employed this technique. The museum buildings are shaded by an iconic shallow dome. "The dome is conceived as a layered series of geometric patterns, superimposed in a manner that appears haptic although it is highly controlled" (Imbert et al. 2012:79). Below the canopy, visible rays of light and dynamic light patterns create a design vision called, "rain of light."

\section{A Comparison Strategy for Different Patterned Shading Screens}

The previous sections provided an overview about how two-dimensional geometric patterns are realized into three-dimensional architectural elements such as flat shading screens, tessellated window frames or domes with superimposed layers of patterns. The next stage of this research is to study the fourth dimension that the 

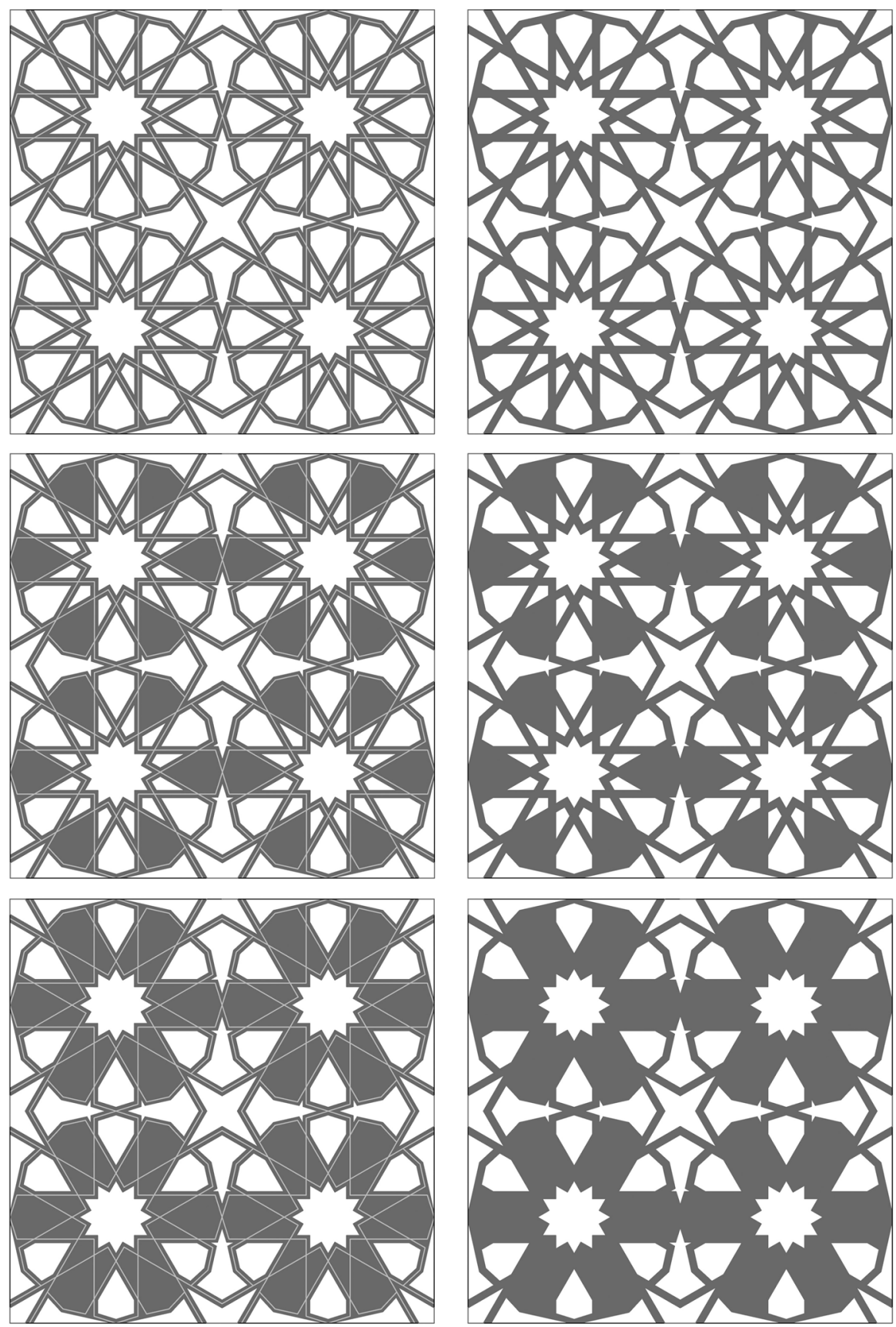

Fig. 16 Different carving patterns, and the visual difference between the patterns with embossed lines (left column) and without embossed lines (right column). Light-gray lines represent the visual effect created by embossed lines 

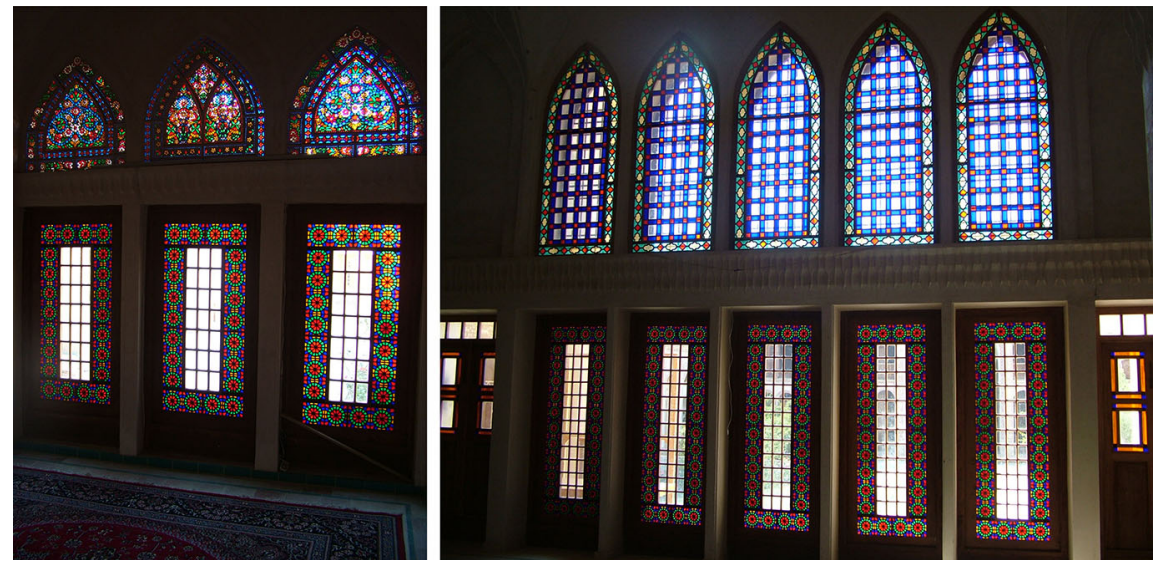

Fig. 17 Tabatayee house, Kashan, Iran

patterned screens bring to architectural spaces. This fourth dimension results from the dynamic play of light and shade in the space and may be addressed from a qualitative or quantitative point of view by using different methods. The qualitative aspects of studying these patterns may include individual preferences related to aesthetics and cultural acceptance also affected by location and purpose, including preferred view to the outside, desired privacy for the inside, the optical distraction of the shading patterns, and productivity of the occupants. The quantitative aspects of studying patterned shading screens include measurement of the acceptable daylight factor, the illuminance, the visual comfort, the annual glare and energy performance. Combining both is also possible by correlating a qualitative factor with a quantitative one.

Several studies have assessed both quantitative and qualitative aspects associated with screens. For example, a group of researchers evaluated how changing the perforation ratio and depth of the screens influences the annual energy loads. Then they defined an optimum depth to perforation ratio for various window orientations (Sherif et al. 2012a). In a study by Francesco Ruggiero and colleagues (2009), the researchers found that the Islamic wall allows a more uniform luminance distribution within a confined environment, which is necessary to create visual comfort conditions. They measured the physiological aspect of vision by measuring the contrast caused by light as well as the psychological aspect of vision, by describing the available view to the outside. Additional research by Iason Konstantzos et al. (2015) attempted to investigate "view clarity" through shading fabrics and shades. They provided sample test offices equipped with different shading products and measured participants' preferences under sunny and cloudy sky conditions. They identified several significance factors impacting view clarity scores including fabric, sky conditions and viewing distance. Finally another group of researchers proposed a new index named "degree of eye opening (DEO)" to indicate glare in sunny climates in the presence of direct sunlight (Garretón et al. 2014). 
There are thus multiple possible ways to compare different shading configurations. This paper seeks to extract key factors regarding screens including perforation ratio (PR), granularity (GR), and morphology (MOR). Then it assesses performance by measuring the illuminance levels by numerical and experimental studies and correlates the key factors with the performance criteria. The question here is "How the Persian patterns relate to simple patterns?" The benefit of simple patterns is that they are designed for calibrating numerical and experimental studies and also for studying the relation of perforation ratio with illuminance. Simple patterns are particularly useful for coarse numerical studies since they have fewer meshes that lead to efficient computational studies. Furthermore, using simple patterns is consistant with modernist approach that emphasizes not employing the exact pattern, but simplifying it, while maintaining the mood and ambiance that someone would get from the original pattern. To address this question, a "predictive model" that associates Persian patterns with simple patterns needs to be described. This predictive model is used to explain how a complex pattern may be translated to an equivalent simple pattern, measured by its total perforation ratio $(\mathbf{P R})$, granularity (GR), and morphology (MOR). Then the "abstraction process" is demonstrated through a case study of a unit pattern and finally a "replication strategy" for that unit is discussed.

\section{A Predictive Model for Patterned Screens}

This section proposes a predictive model to translate complex patterns to simplified patterns. It correlates their key geometric properties within a certain tolerance. The main metrics associated with this predictive model are defined as perforation ratio (PR) and granularity (GR). Perforation ratio is defined as the ratio of the hollowed areas divided by the total area of a screen, and is a number between 0 and 1 . As PR increases, the screen is more perforated, thus the intensity of light that enters the space is higher. Granularity describes the distribution of the perforations. Within a constant PR, there might be only one large hole, representing a $\mathbf{G R}$ of 1, or 100 small holes representing a GR of 100. As GR increases, more holes are present, thus the daylight is more diffused (as opposed to a concentrated light which causes glare). An index termed measure of intensity and diffuseness of light (MIDL) for screens is defined using the aforementioned metrics:

$$
\mathrm{MIDL}=\mathbf{P R} \times \mathbf{G R}
$$

MIDL provides a basis to qualitatively describe the interactive effects of these two parameters and can be calculated for different patterns.

\section{The Abstraction Process of Complex Patterns}

Once there is a basis to qualitatively describe the interactions between $\mathbf{P R}$ and $\mathbf{G R}$, it is possible to describe the abstraction process. For this purpose, two definitions are presented. The term "reference-pattern" is used to describe a distanced view to a pattern as a whole. The term "patch" is used to describe a view to a zoomed-in area of the reference-pattern. For perforated screens that employ Persian geometric 
patterns, the reference-pattern has equally distributed perforations throughout the screen. The reference-patterns can then be observed at different magnifications. As the magnification becomes finer, self-similar patterns may no longer be noticeable and the perforation ratio of a patch may no longer match the perforation ratio of the reference-pattern. In other words, while patterns are self-similar in different magnifications, their morphology is the same. Once the magnified patch loses selfsimilarity with the reference-pattern, its morphology also deviates from the morphology of reference pattern. To capture this effectively, a third parameter of morphology (MOR) is introduced. MOR is qualitatively defined as a number between 0 and 1 , where 1 represents a complete match of two morphologies and a 0 shows no match between the patch and the reference-pattern. Anything between 0 and 1 is a proportional match. An exact quantification method for MOR is beyond the scope of this paper.

The abstraction process is illustrated in Fig. 18 by using a case study inspired by "square-based twelve-pointed star". The upper-row of Fig. 18 represents the magnification action, and the bottom-row represents the abstraction process. As the magnification is increased, you look for the point in which the PR of the patch deviates from the PR of the reference-pattern. For Fig. 18, this happens at magnification $8 \times$. By continuing to zoom in at $8 \times$, the perforation ratio of the patch is $37 \%$ which deviates from the perforation ratio of the reference-pattern which is $41 \%$. The limit of the magnification process is the point at which the PR of the patch no longer matches the PR of the reference-pattern. Therefore, we take

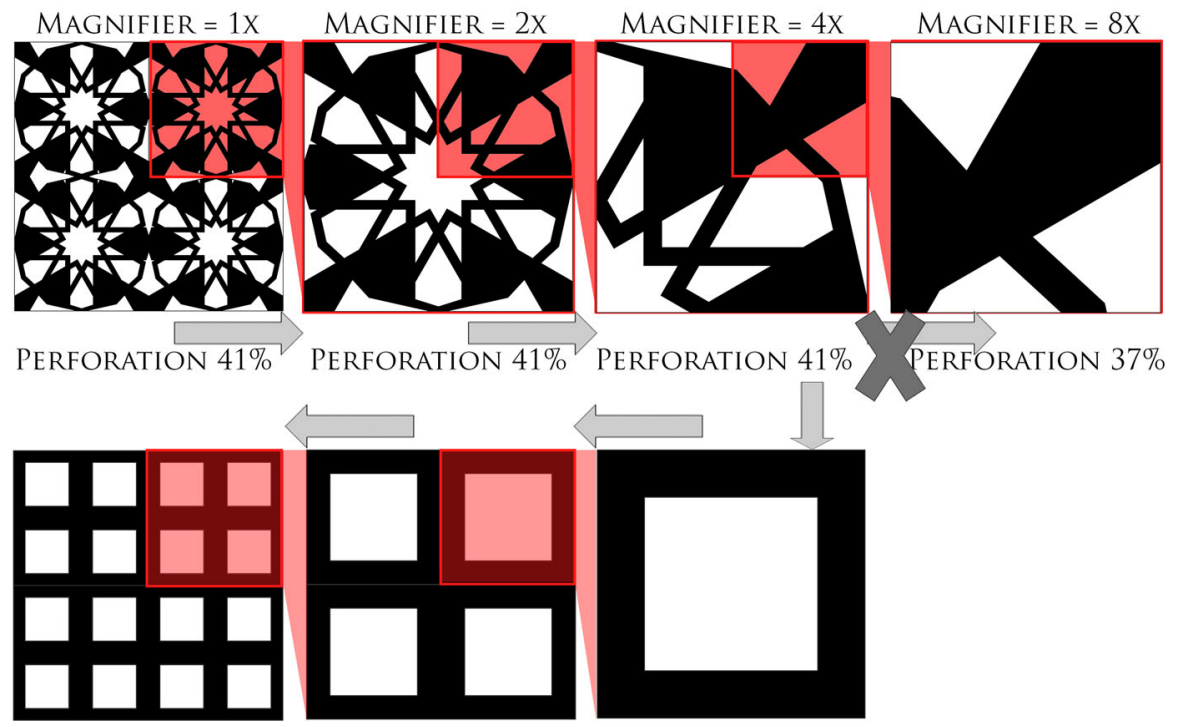

Fig. 18 Upper-row: "magnification process" from left to right. The magnification of the referencepattern is labeled $1 \times$, along with its calculated PR of $41 \%$. Then the magnifier becomes finer showing $2 \times$, while the $41 \% \mathrm{PR}$ is maintained. Next, a finer magnifier of $4 \times$ is shown, while the PR is still maintained at $41 \%$. At magnification of $8 \times$, PR becomes $37 \%$ which deviates from the PR of the reference-pattern. Lower-row: "Abstraction process" from smallest magnification $(4 \times)$ to the largest magnification $(1 \times)$ 
a step back to the last magnification that the perforation ratio of the patch matches the reference-pattern. The appropriate last magnification for Fig. 18 is $4 \times$. This magnification can be used as the starting point for the abstraction process, therefore the area associated in $4 \times$ magnification is used for abstraction.

In the bottom-row of Fig. 18 the area of the scattered apertures in magnification $4 \times$ is calculated and then multiple scattered apertures are replaced with only one aperture maintaining the same area. In this example, all scattered apertures are replaced with one square in the middle. Then moving from right to left, the magnifier zooms out and the abstracted module is replicated through the whole pattern. It should be noted that by employing this method, GR and MOR of the patch largely deviate from the reference-pattern, since multiple apertures are replaced with only one geometrical shape. The $\mathbf{P R}$ however is maintained.

One limitation of replacing the scattered apertures with one square is that the choice of geometric shape is arbitrary and that the abstracted screen may not possess the same visual effects and lighting distributions as the original screen. The impact of this limitation depends on the scale of the apertures and dimensions of the screen. With a fine enough resolution, the abstraction method will still be of use.

In order to develop an abstraction method that resembles the pattern of the original, all three parameters, PR, GR and MOR, can be integrated for pattern abstraction. This process is illustrated in Fig. 19, which is another case study inspired by "square-based twelve-pointed star". Through this process, the magnification starts at $1 \times$ and is carried on until it reaches the smallest self-similar module in the pattern. For Fig. 19 it is magnification $2 \times$. Starting from that resolution, a coarse pattern which roughly corresponds with the perforated pattern is extracted. This coarse pattern strongly depends on geometric families, four-fold, five-fold and six-fold, as discussed by Broug (2013). The abstracted pattern is then replicated throughout the screen. Once the first layer of abstraction has been done, other layers of abstraction can be superimposed on it. As more layers are superimposed, the abstract model resembles the reference pattern more. This process results in PR that fluctuates while various layers of abstraction are being superimposed, however, the MOR and GR become closer to that of the original pattern. The steps taken in this process depend on the equipment and research goals.

\section{The Replication Strategy of an Abstracted Unit}

Once a unit pattern has been abstracted, it requires a strategy to be replicated throughout the whole pattern. The proposed strategy for replication is based on Broug's categorization of different underlying construction grids for patterns (Broug 2013). This approach uses three grid types including a triangular one, a square one and a hexagonal one. This classification can be used as a guide for replicating an abstracted unit pattern. As an example, if a solar screen is designed derived from the "hexagonal based twelve pointed" star (Sarhangi 2012), its abstracted version can be constructed on a hexagonal grid (Fig. 20). 


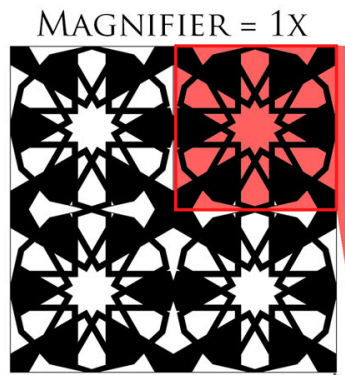

MAGNIFIER $=2 \mathrm{X}$

PERFORATION $41 \%$
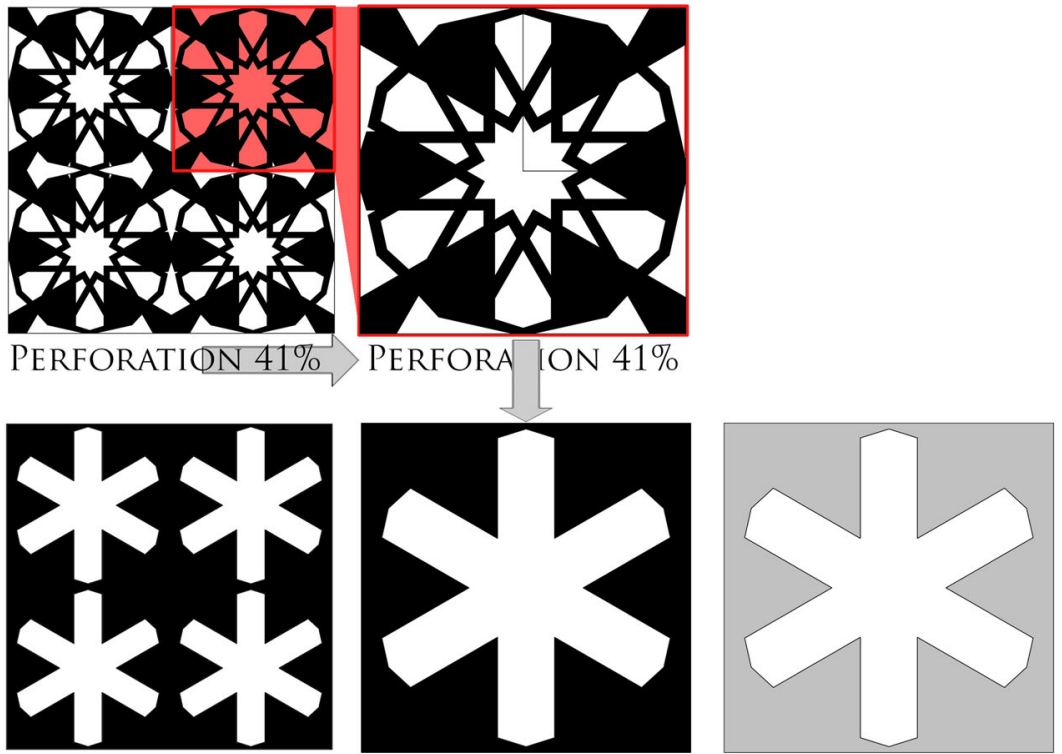

PERFORATION $41 \%$

FIRST ABSTRACTION LAYER
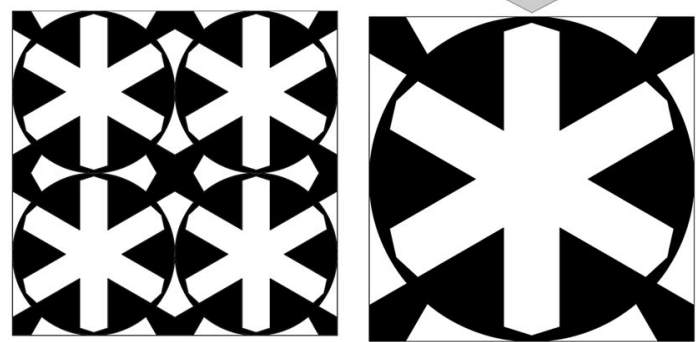

PERFORATION 53\%

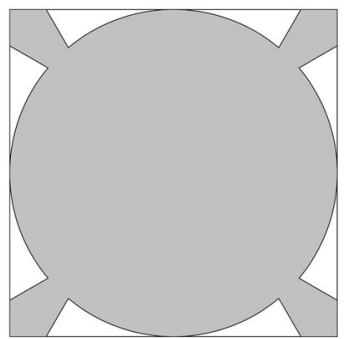

SECOND ABSTRACTION LAYER

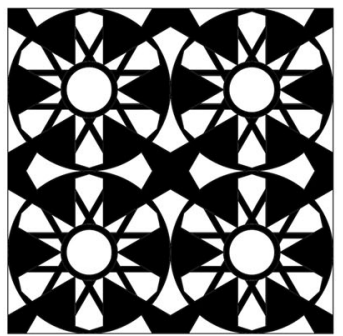

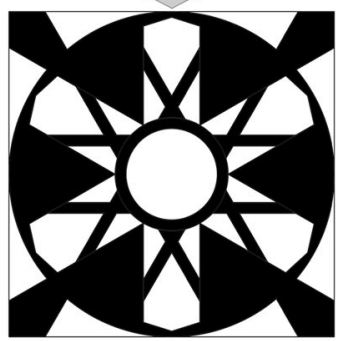

PERFORATION 43\%

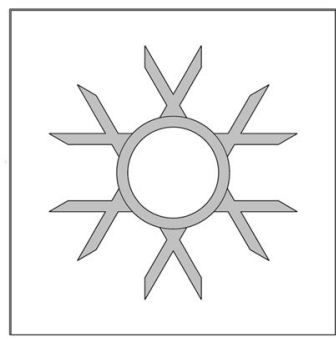

THIRD ABSTRACTION LAYER

Fig. 19 First row from left to right magnification process of reference-pattern starting from $1 \times$ until it reaches the smallest self-similar unit pattern at magnification $2 \times$ The second row (from right to left) extracting a coarse pattern corresponding to the patch, then replicating it. Third and fourth row (from right to left) superimposing layers of detail onto the coarse pattern that is extracted, then replicating it. PR fluctuates from 41 to $53 \%$ and $43 \%$ as additional layers are superimposed on the first layer. 

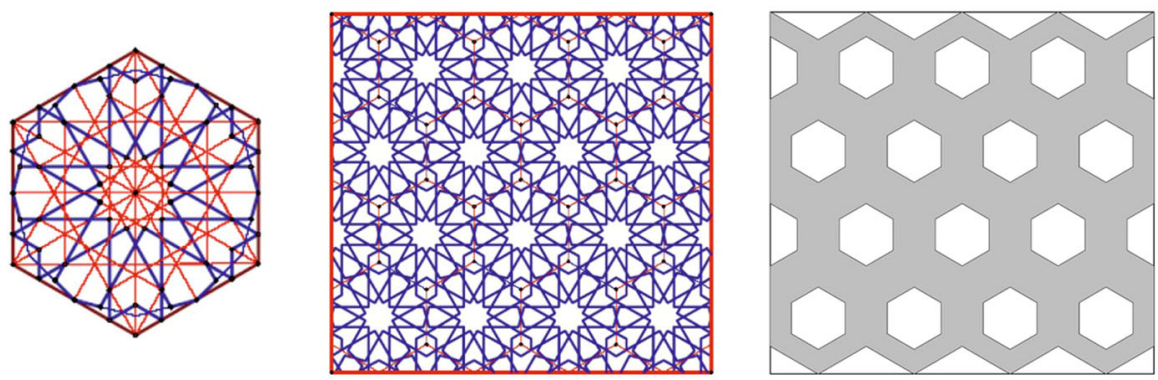

Fig. 20 an abstracted solar screen; left "hexagonal based twelve pointed" star tiling (Photo by Sarhangi 2012, reproduced with kind permission); right the proposed abstracted pattern

\section{Measuring Daylight Performance of Simple Screens: Patterns Under Light and Shade}

This phase of the research consists of numerical and experimental simulations that study how changing the perforation ratio of a shading configuration affects the daylighting performance of space, by observing the relation between perforation ratio PR and illuminance. Once the simulation software is verified with experimental studies, various patterns can be simulated. For this purpose, a few shading screens with simple patterns are designed and are used in a room. The "point in time illumination" on an adjacent wall of the window is measured to compare effects of different patterns. The retrieved results from both strategies are then compared. This method helps to verify the agreement between these two methods for further use of simulation programs in analyzing diverse panel configurations.

\section{General Settings for Daylighting Study}

This section explains the settings for conducting numerical and experimental studies. An apparatus was utilized with the dimensions of $25 \times 15 \times 10$ inches $(0.635 \mathrm{~m} \times 0.381 \mathrm{~m} \times 0.254 \mathrm{~m})$, with a south facing opening of $6 \times 6$ inches $(0.15 \mathrm{~m} \times 0.15 \mathrm{~m})$ in the center of one side. All exterior surfaces were assumed to be black, to decrease the reflectance of the sunlight. Three of the interior surfaces in addition to the ceiling and floor were assumed to be white to achieve the utmost reflective of sunlight inside the space. Other interior walls were simplified to be a black (Fig. 21).

The chosen date for conducting the experimental studies was April 11th, 2014 at solar noon (13:33 daylight saving time) in Ann Arbor, Michigan, with a clear sky and sun. The weather files for Ann Arbor were downloaded from US. Department of Energy website (http://www.energy.gov).

In the first experiment, the relationship between illuminance (the incident light towards the surface) and luminance (the reflected light off the surface) was tested. For this, a white vertical plane was placed in front of the window, and the luminance 


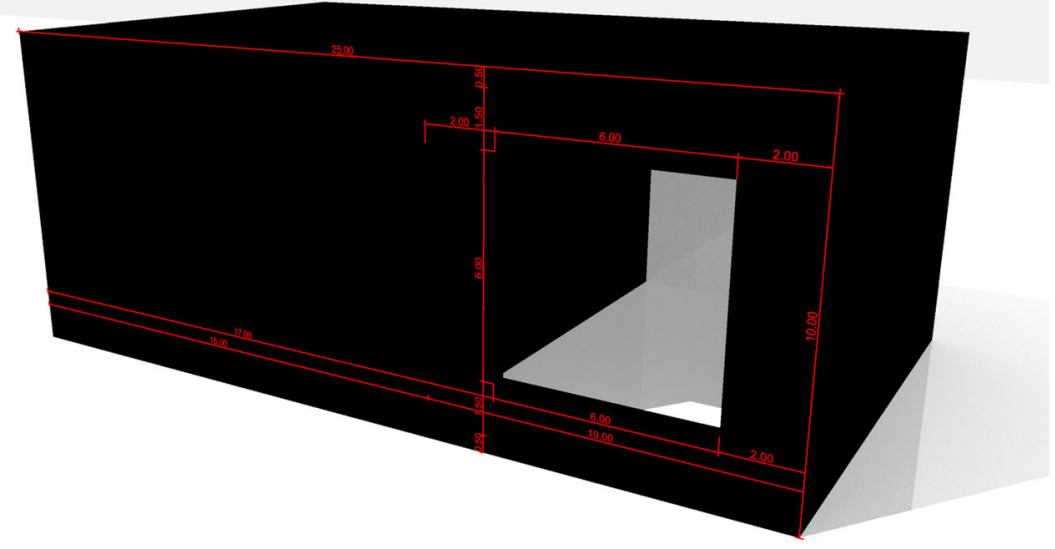

Fig. 21 The modeled box and the dimensions
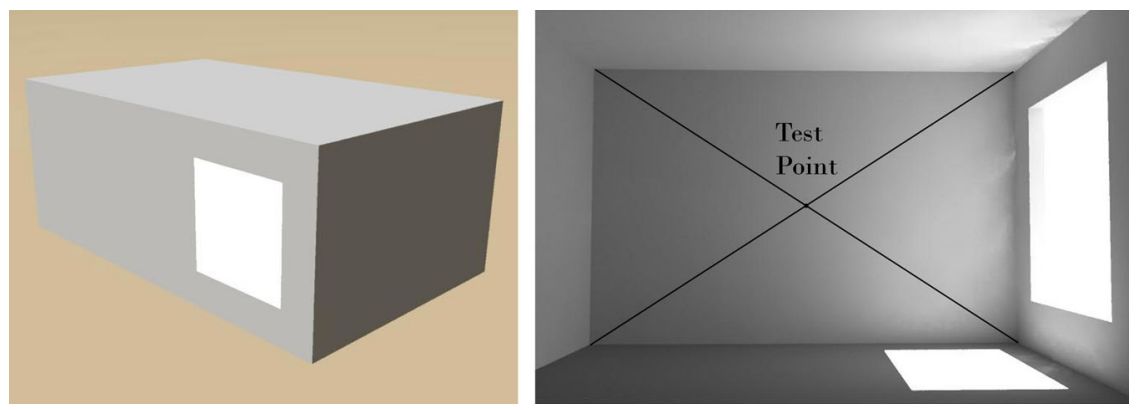

Fig. 22 Exterior and interior of the model; a left measurement on the vertical plane of the window; b right interior test point on the adjacent wall

as well as the illuminance were measured. The purpose of this test was to obtain a ratio between these two indices.

In the second experiment, the point-in-time illumination was measured to compare the performance of different configurations. The reading point has been set in the center of the white wall adjacent to the window (Fig. 22b). This location was selected because it is not exposed to direct sunbeams, and consequently not subject to radical changes of the incoming illuminance. Instead, it provides a measure of the daylighting distribution throughout the room. The reading values are then utilized to calibrate results among simulation and test measurements.

Three different geometries for shading screens have been tested in addition to a case with no shading screen in front of the aperture. The design variations are shown in Table 1. 
Table 1 Experimental study by placing various shading configurations infront of the box and measuring the luminance, and their setup

\begin{tabular}{|c|c|c|c|c|}
\hline $\begin{array}{c}\text { Shading } \\
\text { configuration }\end{array}$ & Case 1: No Shading & Case 2 卌 & Case 3 & Case 4 曲 \\
\hline & & $\begin{array}{c}0.73 \text { " Deep blinds } \\
\text { distanced } 0.67 \text { " from } \\
\text { each other- with glass }\end{array}$ & $\begin{array}{l}\text { A } 24 \% \text { perforated } \\
\text { screen- no glass }\end{array}$ & $\begin{array}{l}\text { A } 43 \% \text { perforated } \\
\text { screen- no glass }\end{array}$ \\
\hline $\begin{array}{c}\text { Placing } \\
\text { shading } \\
\text { configurations } \\
\text { infront of the } \\
\text { aperture }\end{array}$ & & 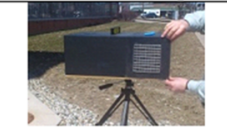 & & \\
\hline $\begin{array}{c}\text { Photo from } \\
\text { the hole } \\
\text { towards the } \\
\text { measurment } \\
\text { point }\end{array}$ & & & & \\
\hline $\begin{array}{l}\text { Measured } \\
\text { Luminance }\end{array}$ & $1800 \mathrm{~cd} / \mathrm{m}^{\wedge} 2$ & $810 \mathrm{~cd} / \mathrm{m}^{\wedge} 2$ & $450 \mathrm{~cd} / \mathrm{m}^{\wedge} 2$ & $830 \mathrm{~cd} / \mathrm{m}^{\wedge} 2$ \\
\hline
\end{tabular}

\section{Field Measurements}

An existing physical box measuring $25 \times 15 \times 10$ inches $(0.635 \mathrm{~m} \times 0.381 \mathrm{~m} \times$ $0.254 \mathrm{~m})$, with a south-facing opening of $6 \times 6$ inches $(0.15 \mathrm{~m} \times 0.15 \mathrm{~m})$ was used to detect light transmittance through different shading configurations. This model was attached to a tripod and balanced with the aid of a level. Then, the accurate position and direction of the box was confirmed by utilizing a compass. The test was conducted on a sunny day on April 11th, 2014, which simulates the clear sky condition with sun. The shading configurations were placed to cover the $6^{\prime \prime} \times 6^{\prime \prime}$ opening. The meter, Sekonic L-758C, was used to measure the illuminance and luminance levels (Fig. 23).

Incident and reflected light were metered. The reflected metering measured light that was reflected off a surface. The incident metering measured light that was falling on a surface. To measure the reflected light, the light meter was pointed directly at the object's position (Fig. 24a). To measure the incident light the light meter was held in front of the subject and pointed towards the light source (Fig. 24b).

The first experiment measured the incident light falling on the surface and the reflected light off the surafce. First, a white plane was placed in front of the windowand the amount of incident and reflected light was measured. The incident light towards the surface was 70,000 lux (illuminance) and the reflected light off the surface was $23,000 \mathrm{~cd} / \mathrm{m}^{2}$ (luminance). There was a ratio of around 3.043 between illuminance and luminance on the white plane. In the second experiment, each shading configuration was placed in front of the aperture (Fig. 25a). Using the circular hole in the scaled box (Fig. 25b), the reflected light off the middle point of the interior wall was measured. The light measurements for each configuration are 

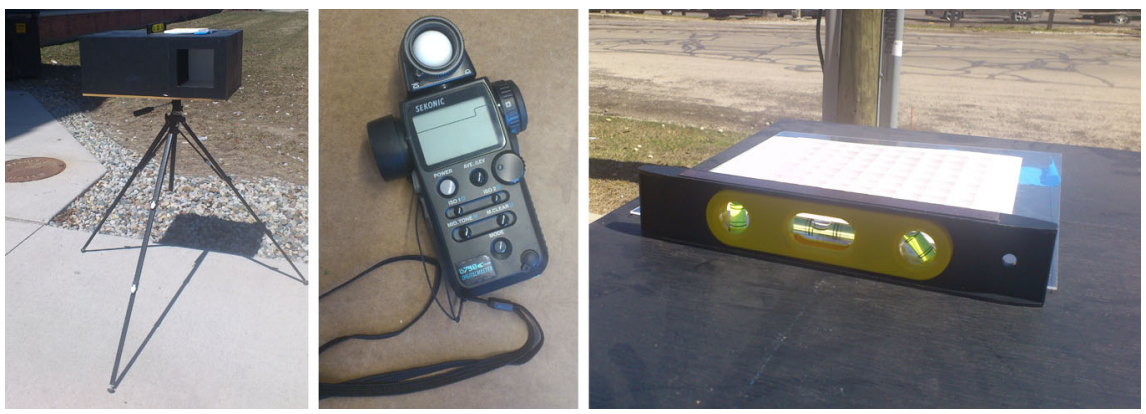

Fig. 23 The physical measurements setup; left the box attached to the tripod; middle the lighting meter; right the level
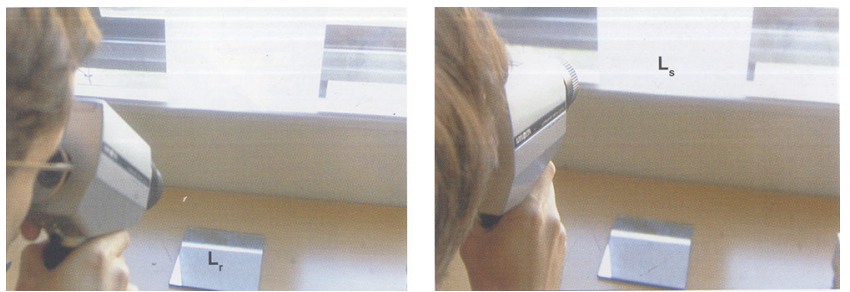

Fig. 24 a Left Measuring the luminance of reflected light off a surface; b right measuring the illuminance of the source of the light towards that surface (Fontoyont 1999)
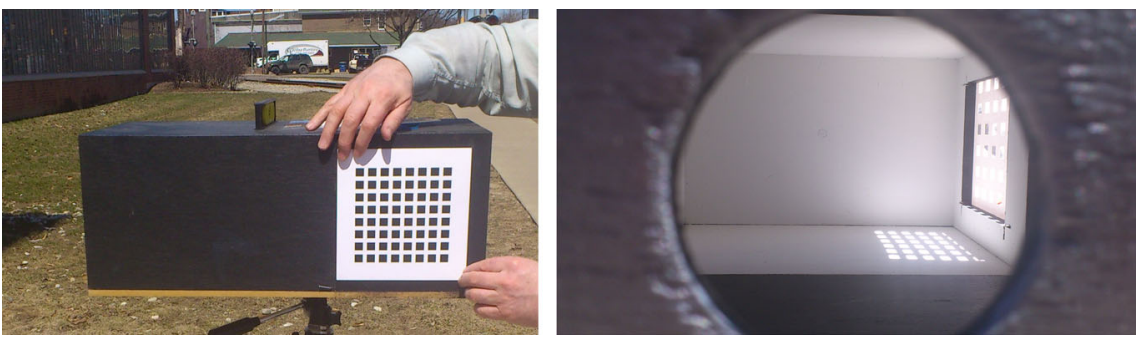

Fig. 25 Experimental process; a left placing the shading configuration; b right measuring the reflected light through the hole carved in the scaled box

presented in Table 1. Except for case 2 which had a layer of acrylic attached to the blinds, all other configurations were simulated as a screen without an added layer of glass.

\section{Numerical Simulations: DIVA}

Regarding the computational studies, Rhinoceros-NURBS modeler was used as the modeling platform with DIVA-for-Rhino plugin that allows for highly optimized daylighting and energy modeling. The simulations are based on powerful environmental performance engines such as Radiance, Daysim and Energy Plus 
Table 2 Final DIVA for Rhino settings

\begin{tabular}{ll}
\hline Location & Ann Arbor, Michigan \\
\hline Date & April 11th, 2014 \\
Time & solar noon (13:33 daylight-saving time = 12.6 for DIVA) \\
Materials & "Generic ceiling (80)" assigned to all white surfaces \\
& "Generic Floor (20)" assigned to black walls \\
& "Outside Ground (20)" assigned to outside ground \\
& "Single pane glazing (88)" assigned to glazing \\
Sky-types & "Outside façade (35)" assigned to shading screen \\
Setting parameters & Clear sky with the sun \\
& ab 5,ad 1500, as 20, ar 300 \\
& The grid size was 0.5 at 0.1 " distance from the wall.
\end{tabular}

${ }^{a}$ The default materials in DIVA for Rhino

(Jakubiec et al. 2011). The simulation process in DIVA started with a set of basic assumptions about the materials by assigning the default materials to the wall surfaces. The outcomes of the simulation study were compared to the results of the experimental study, and the materials were being altered one at a time, to obtain the closest results with the experiment. Finally, a material setting that yielded the closest outcome compared with experimental studies was recorded (Table 2). The simulation results are illustrated in Table 3.

\section{Flamingo nXt Simulations}

Regarding the computational studies, a second software was also used for the numerical studies. Flamingo $\mathrm{nXt}$ is a renderer engine for Rhino, which uses a method of multi-pass refinement. It can place a range of lights on different channels, which can be edited later without the need to re-render the scene. Flamingo $\mathrm{nXt}$ produces realistic results by simulating light transport in the model. Indirect lighting, as well as complex reflection and refraction, can be incorporated into the simulation. After the simulation is completed, the image can be saved as a "native Flamingo nXt file". This file can be browsed and opened in the Rhino scene while the user can detect the luminance values by hovering the mouse over the desired points. Using Flamingo, the luminance value can be detected with the different material setting. For the simulation, "Pure matt", "black matt", "grey dark matt", "basic clear glass" and "pure matt" were assigned to all white surfaces, black walls, outside ground, glazing and shading screens respectively. The date and time matched the first simulation. These values are plotted along with the experimental results. The material and sky settings that lead to result close to the experimental outcomes were opted for future simulations.

\section{Calibration Results}

To calibrate results, output from DIVA and in Flamingo were compared with the experimental results. The comparison is plotted in Fig. 26 and show good 
Table 3 A Simulation study, rendering, luminance false-color image and the illuminance grid

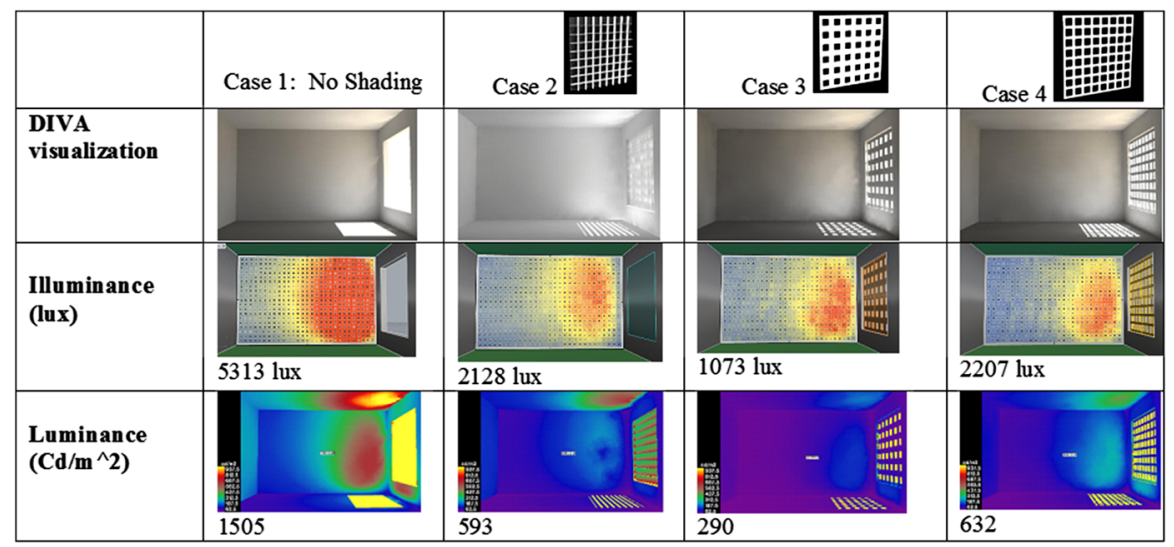

agreement with measurements and DIVA simulation tool. The verification provided a ground to employ simulation for further studies. Simulation studies are faster and less expensive, and they provide advantages as Reinhart (2014) points out: "Although scale models are superior to simulation-based exploration in terms of accuracy, but simulations are less expensive and save on time in terms of constructing models" (Reinhart 2014:182). It is expected that the results provide a robust platform to conduct further analysis using these programs. This calibration also aids in better understanding the accuracy of the programs, as well as helps identify their positive and negative features (Sun et al. 2014).

\section{Numerical Study of a Screen Inspired by Persian Patterns}

Once the simulation results were verified through experimental studies, various shading screens were simulated to assess their performance. A shading screen inspired by the "square-based twelve-pointed star", illustrated in Fig. 17 was designed and modeled. The perforation ratio of this screen was $41 \%$, which is very close to case study 3 (see Tables 1,3). Maintaining the same perforation ratio, the resolution of the pattern was varied to create four different shading configurations (Fig. 27). It should be noted that the embossed lines were not modeled here since the perforation ratio, and the granularity of a pattern are not related to the presence of the embossed lines. The visualization and the false color rendering of the screen are presented in Table 4.

The point in time illuminance was plotted for screens with different granularities, along with a yearly metric namely daylight autonomy (DA) (Fig. 28) which is defined as the "percentage of the occupied times of the year when the minimum illuminance requirement at the sensor is met by daylight alone" (Reinhart et al. 2006). In this research, DA was calculated throughout a year from 8 AM to 6 PM weekdays and was set to 500 lux to satisfy the minimum daylight level for an office. 


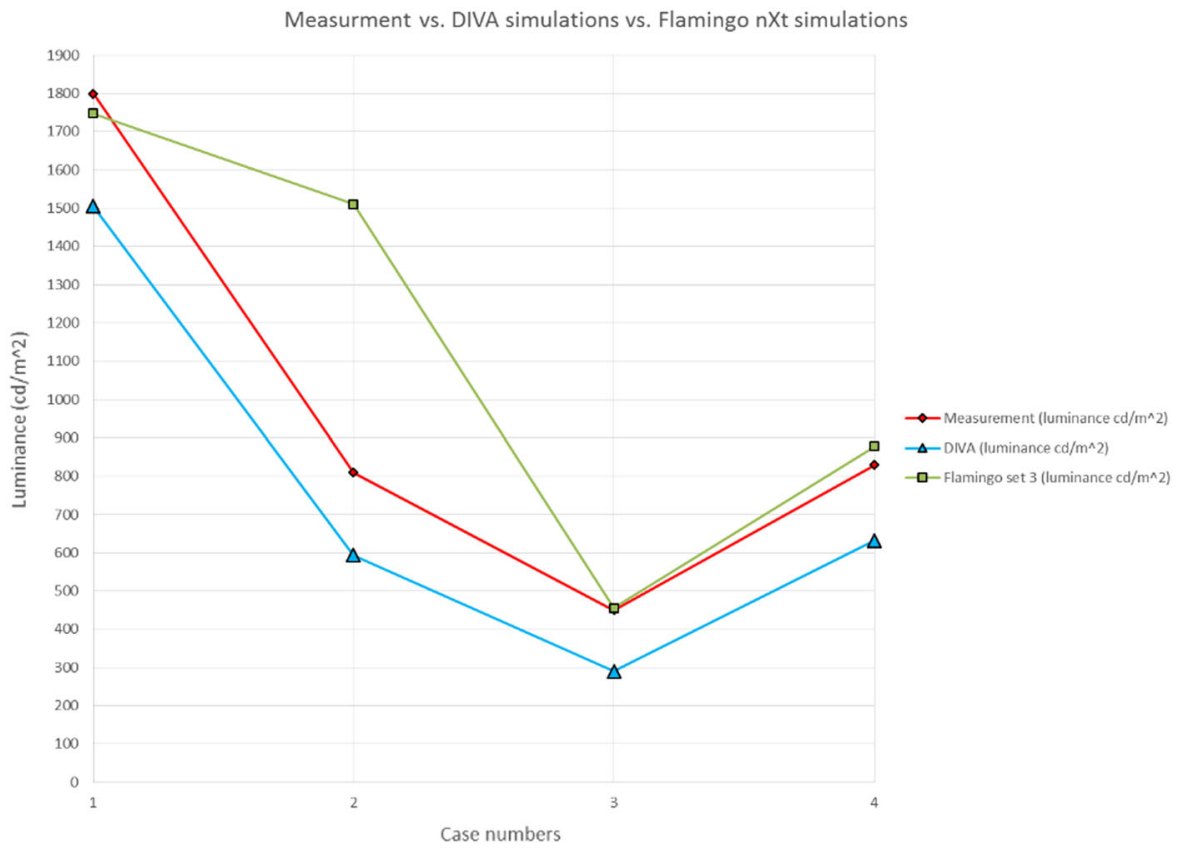

Fig. 26 The final comparison between experimental measurements, DIVA plugin, and Flamingo $\mathrm{nXt}$ simulations. The plot demonstrates a good agreement among them
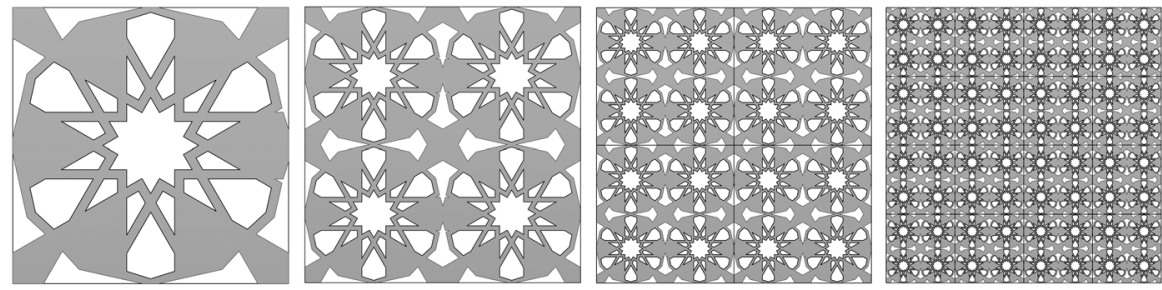

Fig. 27 a shading screen in different resolutions represents different GRs within a constant PR

Therefore, if space with 500 lux requirement had a DA of $80 \%$ for one sensor, it means that the required lighting level would be met by daylight alone during $80 \%$ of the year for that sensor. Artificial lighting can then be used to make up the remaining $20 \%$. The horizontal DA was measured on a horizontal set of nodes which was placed at desk level of 0.9 meters.

As the pattern becomes finer, the illumination on the vertical wall as well as the daylight Autonomy decreases (Fig. 28). It is important to note that the perforation ratio is the same in all cases, and they are only scaled down. This study reveals that in the study of shading screens, it is important to consider the size of the apertures, as well as the overall perforation ratio. 
Table 4 A Simulation study, rendering, luminance false-color image
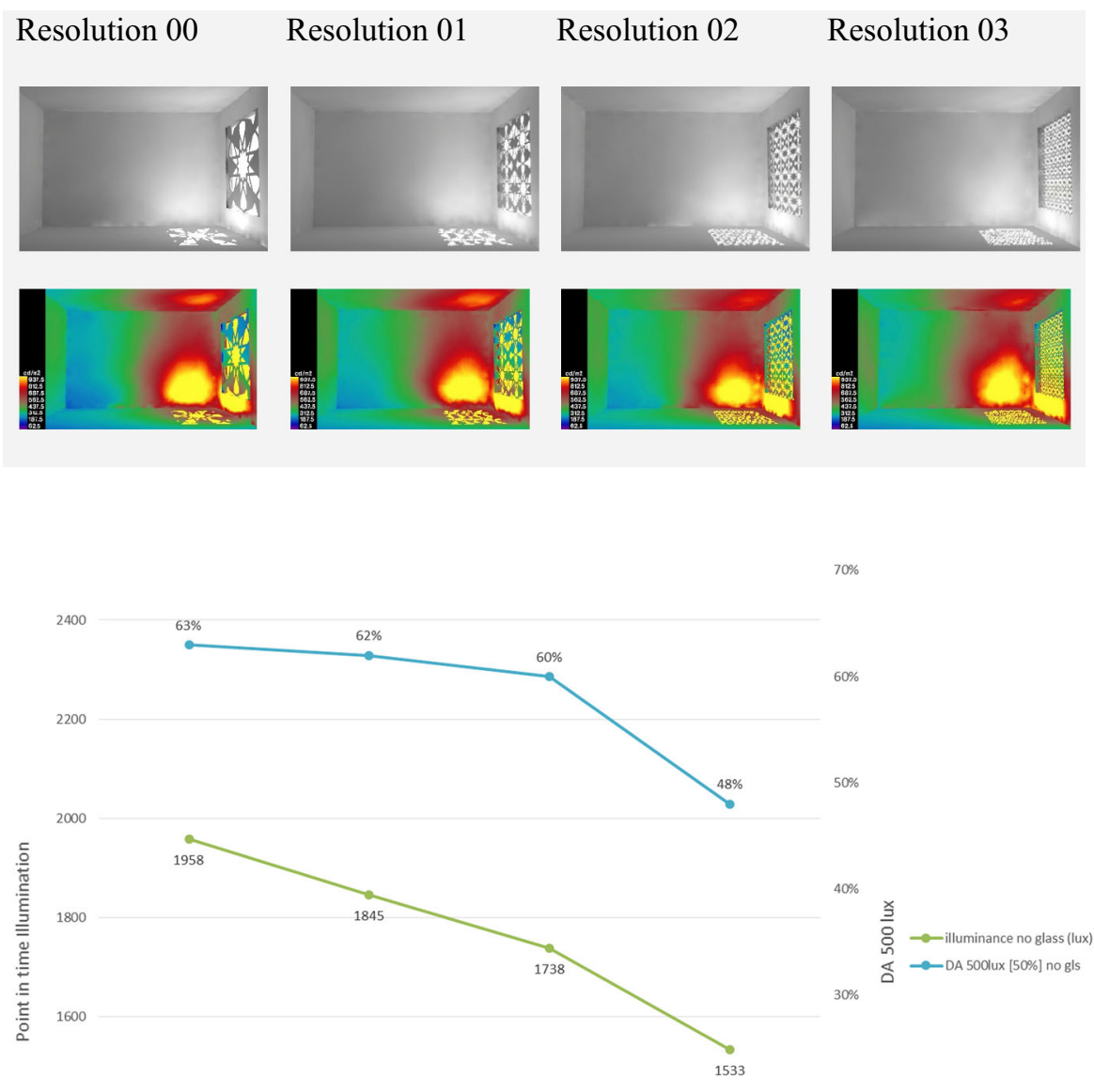

$\varangle \rightarrow$ DA 500lux [50\%] no gls

1400

$20 \%$

1200

Fig. 28 Point in time illuminance and DA of different screens

\section{Conclusion}

Geometric patterns have evolved from simple patterns into more complex ones over time, perhaps due to improvements in material and construction technologies. The advances in complex patterns are also a function of increased experiences and accumulation of lessons learned from previous experiences. This process ultimately builds up the knowledgebase for delivering complex patterns. Geometric patterns can be applied to shading screens for architectural spaces, not only as an ornamental 
feature but also as an environmental control system that modulates daylighting. In this study, the mathematical rules behind constructing the patterns was reviewed. Then, different methods of realization with various materials was studied. This was accomplished to assess how these complex patterns compared with simple ones, by proposing a "predictive model." The reason for emphasizing simple patterns in the study of patterned shading screens was twofold. First, the simplified patterns can be very useful for a coarse computational study since a simple geometry has fewer meshes and is computationally more efficient. Second, a modernist approach in using these patterns in different contexts suggests not always to use the exact pattern, but to simplify it, while maintaining the mood and ambiance of the original pattern. This predictive model employs three main parameters: Perforation ratio, Granularity and Morphology. Once this predictive model and its framework was defined, a simulation and experimental study was conducted that assessed the lighting performance of some simple shading screens. The results of the study showed agreement between experimental and numerical studies. Also, it was demonstrated that as the perforation ratio decreased, the lighting levels decreased as well. This provided a good measure for PR, but not GR nor MOR. Four instances of one shading screen inspired by Persian patterns were then simulated. These patterns had same perforation ratio, but different granularities. The results of the simulations illustrated that perforation ratio is not the only factor that influences the lighting performance, but that the size of the apertures within the same perforation ratio also affected the daylighting performance of the buildings. This aspect can be studied further by looking at how the size of the apertures affects glare, which help to reduce glare problems.

It should be noted that the proposed predictive model is based on studying very few geometric patterns and requires further investigation on how it may encompass varieties of patterns to turn it into a comprehensive model. Further research is needed to quantify better the metrics of, PR, GR and MOR. This study will be of value to architects who design patterned shading screen, by informing their decisions about the most appropriate way to assess possible designs' performance.

Acknowledgments All photos and images are by Niloufar Emami unless otherwise indicated.

\section{References}

Abas, Syed Jan, and Amer Shaker Salman. 1992. Geometric and Group-Theoretic Methods for Computer Graphic Studies of Islamic Symmetric Patterns. Computer Graphics Forum 11 (1): 43-53.

Broug, Eric. 2013. Islamic Geometric Design. New York: Thames \& Hudson Ltd.

Cenani, Sehnaz, and G Cagdas. 2007. A Shape Grammar Study: Form Generation with Geometric Islamic Patterns. In: Proceedings of 10th Generative Art Conference, pp 1-7.

Emami, Niloufar, Anahita Khodadadi, and Peter von Buelow. 2014. Design of Shading Screen Inspired by Persian Geometric Patterns: An Integrated Structural and Daylighting Performance Evaluation. In: IASS (International Association for Spatial and Shell Structures). Brasilia, Brazil

Fontoyont, Marc. 1999. Daylight Performance of Buildings. London: James \& James.

Gelil, Nermine Abdel. 2006. A New Mashrabiyya for Contemporary Cairo: Integrating Traditional Latticework from Islamic and Japanese Cultures. Journal of Asian Architecture and Building Engineering 5(1):37-44 
Imbert, Frédéric, KathrynStutts Frost, Al Fisher, Andrew Witt, Vincent Tourre, and Benjamin Koren. 2012. Concurrent Geometric, Structural and Environmental Design: Louvre Abu Dhabi. In: Advances in Architectural Geometry, edited by Lars Hesselgren, Shrikant Sharma, Johannes Wallner, Niccolo Baldassini, Philippe Bompas, and Jacques Raynaud, pp 77-90. Springer-Verlag/ Wien.

Jakubiec, Alstan, and Christoph Reinhart. 2011. Diva 2. 0 : Integrating Daylight and Thermal Simulations Using Rhinoceros 3D, Daysim and Energyplus. In: Proceedings of Building Simulation, pp 2202-9.

Kaplan, C S. 2000. Computer Generated Islamic Star Patterns. In: Third Annual Bridges Conference, pp 105-12.

Konstantzos, Iason, Ying-Chieh Chan, Julia C. Seibold, Athanasios Tzempelikos, Robert W. Proctor, and J. Brent Protzman. 2015. View Clarity Index: A New Metric to Evaluate Clarity of View Through Window Shades. Building and Environment 90:206-214

Reinhart, Christoph. 2014. Daylighting Handbook VolumeI: Fundamentals Designing with the Sun. Edited by Ria Stein. Publisher not identified.

Reinhart, F, Christoph., John. Mardaljevic, and Zack. Rogers. 2006. Dynamic Daylight Performance Metrics for Sustainable Building Design. In LEUKOS, 3:7-31.

Ruggiero, Francesco, Rafael Serra Florensa, and Antonella Dimundo. 2009. Re-interpretation of traditional architecture for visual comfort. Buildings and Environment 44(9): 1886-1891

Sarhangi, Reza. 2012. Interlocking Star Polygons in Persian Architecture: The Special Case of the Decagram in Mosaic Designs. Nexus Network Journal 14 (2): 345-72.

Sherif, A., A. El-Zafarany, and R. Arafa. 2012. External Perforated Window Solar Screens: The Effect of Screen Depth and Perforation Ratio on Energy Performance in Extreme Desert Environments. Energy and Buildings 52: 1-10.

Sherif, Ahmed, Hanan Sabry, and Tarek Rakha. 2012. External Perforated Solar Screens for Daylighting in Residential Desert Buildings: Identification of Minimum Perforation Percentages. Solar Energy 86 (6): 1929-40.

Sun, Chanjuan, Harry Giles, and Zhiwei Lian. 2014. The Dynamic Impact of Window Characteristics on Shading Factor and Energy Consumption. Solar Energy 102:1-13.

Yamin Garretón, Julieta, Roberto Rodriguez, Ana Ruiz, and Andrea Pattini. 2014. Degree of Eye Opening: A New Discomfort Glare Indicator. Building and Environment 88: 142-50.

Niloufar Emami is a Ph.D. Candidate at Taubman College of Architecture and Urban Planning at the University of Michigan. With an architectural background, she has research experience with numerical and experimental studies of structural and material systems, as well as daylighting and energy analysis of building envelopes. Her research interest is in integrated and performance-based design of buildings considering structural and environmental performance, parametric design, multi-criteria decision making in design and application of geometric patterns in structure and architecture.

Harry Giles is Professor of Practice and a designer, researcher and entrepreneur, specializing in structural engineering, integrated building and product design. He teaches graduate courses in advanced materials and geometrically complex surface structures. He has over 30 years of consulting experience in cutting edge building design innovation as a principal in Arup and Buro Happold in Africa, Europe, Turkey and USA. His passion is in integrated interdisciplinary design, and building product commercialization. He has been the Principal Investigator on various NSF, EPA and university funded research projects in the USA and China. Harry is also a principle of SITUMBRA LLC and solartonic llc, which designs and manufactures products for the building industry, such as a modular steel prefabricated multi-story housing system, structurally composite transparent façade systems as well as solar lighting and building integrated photo-voltaic technology, with various patents pending. 